\title{
Pesquisa
}

\section{Abordagem da Disciplina de PNE nos Currículos de Odontologia das Instituições de Ensino Superior do Nordeste do Brasil}

\author{
Approach of PNE Discipline in Dentistry Curriculum of Higher Education Institutions \\ of Northeast Brazil
}

Susana Mairlla de Sousa Martins ${ }^{1}$; Clenia Emanuela de Sousa Andrade ${ }^{1}$; Gérsica Thaís Santos de Andrade2; Gustavo Gomes Agripino3; Naiana Braga da Silva ${ }^{3}$; Andreia Medeiros Rodrigues Cardoso ${ }^{4}$.

${ }^{1}$ Acadêmica do Curso de Odontologia, Universidade Estadual da Paraíba, Araruna - PB, Brasil. ${ }^{2}$ Cirurgiã-Dentista, Universidade Estadual da Paraíba, Araruna-PB, Brasil.

${ }^{3}$ Professor no curso de Odontologia da Universidade Estadual da Paraíba, Araruna - PB, Brasil.

${ }^{4}$ Professora no Centro Universitário de João Pessoa, João Pessoa-PB, Brasil.

Autor e endereço para correspondência:

Naiana Braga da Silva, Rua Francisco Chagas de Almeida, 102, Bancários, João Pessoa-PB, CEP:58051-500, e-mail:neinabs@hotmail.com

\begin{abstract}
Resumo
Introdução: Pacientes com necessidades especiais (PNE) necessitam de atendimento especializado e os profissionais de saúde precisam ter esse conhecimento ainda na graduação. Com isso, é de considerável importância existir a disciplina de PNE nos cursos de Odontologia. Objetivo: Apresentar o panorama da abordagem da disciplina de PNE nos currículos dos cursos de Odontologia, no Nordeste do Brasil. Metodologia: Este estudo é do tipo documental quantitativo com análise descritiva onde por meio de uma busca realizada na internet no site do MEC e das 101 Instituições de Ensino Superior (IES) com o curso de odontologia no Nordeste, onde no site de todas as IES cadastradas foi buscado suas grades curriculares e ementas e analisadas de forma quantitativa. As instituições foram avaliadas sobre: a presença ou ausência de disciplina exclusiva para PNE apresentado em seu currículo; a forma de oferta; e as disciplinas afins. Resultados: A maior parte dos currículos das instituições estava disponível para acesso livre. Verificou-se que $75,2 \%$ das IES do Nordeste disponibilizam matriz e/ou ementa via internet. Observou-se que dos currículos disponíveis,44,7\% das instituições apresentam componente específico de PNE. Desta parcela, $91,1 \%$ ofereciam-na como obrigatória e $8,8 \%$ como eletivas e $21 \%$ das IES com matriz/ementa disponível apresentam disciplinas afins. Conclusões: Menos da metade das IES do Nordeste brasileiro oferecem uma disciplina no curso de Odontologia exclusiva para PNE e m relação ao modo de ofertar o componente, predominou o obrigatório.
\end{abstract}

Descritores: Deficiência. Instituições de Ensino Superior. Odontologia. 


\begin{abstract}
Introduction: Patients with special needs (PNE) need specialized care, and health professionals need to have this knowledge in the undergraduate course. With this, it is of considerable importance to have the discipline of PNE in the courses of Dentistry. Objective: To present an overview of the PNE discipline approach in the curricula of Dentistry courses in Northeast Brazil. Methodology: This study is a documentary quantitative study with a descriptive analysis where through a search conducted on the Internet of the MEC website and the 101 Institutions of Higher Education (IES) with the dentistry course in the Northeast, where on the website of all HEls registered was searched its curricular grades and menus and analyzed in quantitative form. The instiuições were evaluated on the presence or absence of exclusive discipline for PNE presented in its curriculum; the form of supply; and related disciplines. Results: Most of the curricula of the institutions were available for free access. It was verified that $75.2 \%$ of the HEls in the Northeast provided a matrix and / or a web page. It was observed that of the available curricula, $44.7 \%$ of the institutions have a specific PNE component. Of this share, $91.1 \%$ offered it as mandatory and $8.8 \%$ as elective and $21 \%$ of HEls with available matrix / menu present similar disciplines. Conclusions: Less than half of HEls in the Northeast of Brazil offer a course in Dentistry exclusively for PNE and in relation to the way of offering the component, the compulsory one predominates.
\end{abstract}

Keywords: Disability; Higher education institutions; Dentistry.

\title{
Introdução
}

Todo ser que necessite de um tratamento especial por causa de uma situação diferente ou variação (ou um conjunto), que seja temporário ou indefinido, com origem fisiológica, morfológica, mental, estado psicológico e mudança comportamental, é considerado PNE e devem ter acesso a todos os serviços de saúde, desde os níveis de atenção básica aos especializados e alta complexidade'.

No ano de 2010, o IBGE (Instituto Brasileiro de Geografia e Estatística) constatou que exitiam aproximadamente 64.380 .132 brasileiros portadores de alguma deficiência visual, auditiva, motora ou mental, equivalente a quase $1 / 4$ da população, sendo a região Nordeste portadora de uma média ainda maior, mais de $26 \%{ }^{2}$.

Os PNEs precisam ser acompanhados por várias especialidades, mesmo que não estejam diretamente relacionadas à sua condição, eles estão sujeitos ao aparecimento de outras doenças ou sofrer agravos caso não aja o cuidado e isso inclui os serviços odontológicos e a atenção à saúde oral. A diferença que existe no atendimento de PNEs, está relacionada ao manejo para 
com o indivíduo e responsáveis, na estrutura arquitetônica e na relação das afecções orais com o resto do corpo, pois a técnica do procedimento são iguais ${ }^{3,4}$.

Alguns PNEs apresentam os certos movimentos limitados e/ou involuntários o que torna difícil a sua própria higiene oral, ou então não têm boa recepção a esse afazer, quando somado essas limitações aos medicamentos usados aumentam o risco de infecções devido uma dieta cariogênica e algumas vezes, torna o atendimento odontológico ainda mais imprescindível, tornando o protocolo convencional da manutenção a cada 06 meses insuficiente diante tais circunstâncias².

Ao analisarem a experiência de pacientes PNE atendidos em um projeto de extensâo do curso de Odontologia da Universidade Luterana do Brasil, Canoas (Rio Grande do Sul), concluíram que se pode solucionar a maior parte das necessidades desses pacientes apenas a nível ambulatorial, podendo realizar os atendimentos desde uma aplicação tópica de flúor (ATF) até a confecção de próteses, e afirma que as maiores dificuldades do atendimento são em relação ao manejo ${ }^{5}$.

Os cirurgiões dentistas (CDs) e os responsáveis por promover a saúde, precisam ser qualificados e capacitados, durante a graduação, a identificar as diferenças e excepcionalidades de todos, para melhor assistir a importante parcela da população que requer cuidados especiais $^{6,7}$.

O ensino universitário molda O CD para ser mais flexível social, psicológica e teoricamente, e o torna mais humano, este ensino deve derrubar os muros, e ampliar os conhecimentos e a possibilidade de atuação, empoderando o aluno a sair da instituição capaz de solucionar os problemas que a população apresentar de frente a seus recursos materiais ${ }^{6,8,9} .0$ componente curricular PNE na Odontologia, nas IESs do Nordeste em geral, era baixo em 2013 e tem sido ainda insuficiente quando comparado a quantidade de pacientes que necessitam 0 atendimento.

Objetivando analisar se alguma mudança ocorreu nos últimos anos, o presente trabalho apresentará o panorama atual da disciplina de PNE, nos cursos de Odontologia das Instituições de Ensino Superior no Nordeste do Brasil ${ }^{10}$.

\section{Metodologia}

Este estudo é do tipo documental quantitativo com análise descritiva. A pesquisa foi realizada no período de fevereiro e março de 2018. Foram admitidas todas as IES cadastradas 
segundo o e-MEC, a base de dados oficial e única de informações relativas à Instituições de Educação Superior e cursos de graduação do Sistema Federal de Ensino, com cursos de Odontologia na região Nordeste, incluindo as matrizes e ementas curriculares das mesmas, sem critérios de exclusão.

A presente pesquisa foi realizada seguindo etapas subsequenciais, a começar pela localização das IES que oferecem o curso de Odontologia, em cada estado nordestino, através do endereço virtual do MEC (www.emec.mec.gov.br). Depois de localizar as instituições, foi iniciada uma busca para aquisição das matrizes curriculares e/ou ementa do curso, através dos sites oficiais das instituições cadastradas.

Após isso, as IESs foram classificadas segundo a oferta de disciplina exclusiva de Odontologia para PNEs e as disciplinas afins que são componentes onde são abordados temas que envolvem alguns tópicos de PNE, encontrado em suas respectivas matrizes curriculares. Foi feita uma análise quantitativa do total de instituições, por estado, que oferecem o componente, que não oferecem, as que oferecem disciplina afins e as com matriz indisponível.

E por fim, as instituições com ementa disponível foram analisadas de forma quantitativa em relação ao modo de oferta, se obrigatório ou eletivo; quanto ao sistema de abordagem da disciplina, apenas teórica, apenas prática ou teórico-prática; e as disciplinas afins. O fluxograma a seguir mostra a sequência aplicada (Figura 1). As informações obtidas em cada etapa eram analisadas estatísticamente de forma descritiva.

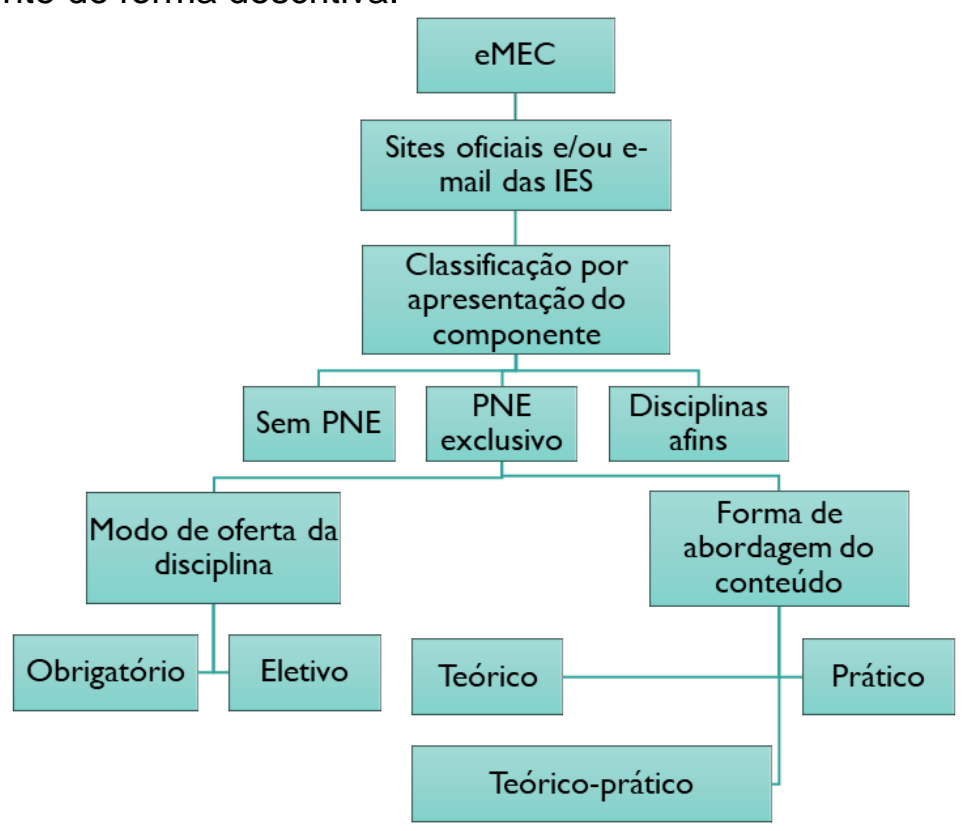

Figura 1. Fluxograma de etapas aplicadas à metodologia. 


\section{Resultados}

A maior parte dos currículos das instituições estava disponível para acesso livre. Foram encontradas 101 escolas de graduação com curso de Odontologia nos estados do Nordeste, no Cadastro e-MEC de Instituições e Cursos de Ensino Superior.

Aparecem Bahia, Pernambuco e Paraíba como os Estados do Nordeste com mais instituições cadastradas no MEC, (destacando 32, 14 e 13 respectivamente) com curso de graduação em Odontologia.

Do total, 76 escolas $(75,2 \%)$ disponibilizaram suas grades curriculares nos sites oficiais, contra $25(24,7 \%)$ que não estavam livres para acesso. Com os currículos acessíveis foi possível observar quantas instituições concedem ao menos uma disciplina exclusiva de Odontologia para PNE e as que apresentam disciplinas afins (Figura 2).

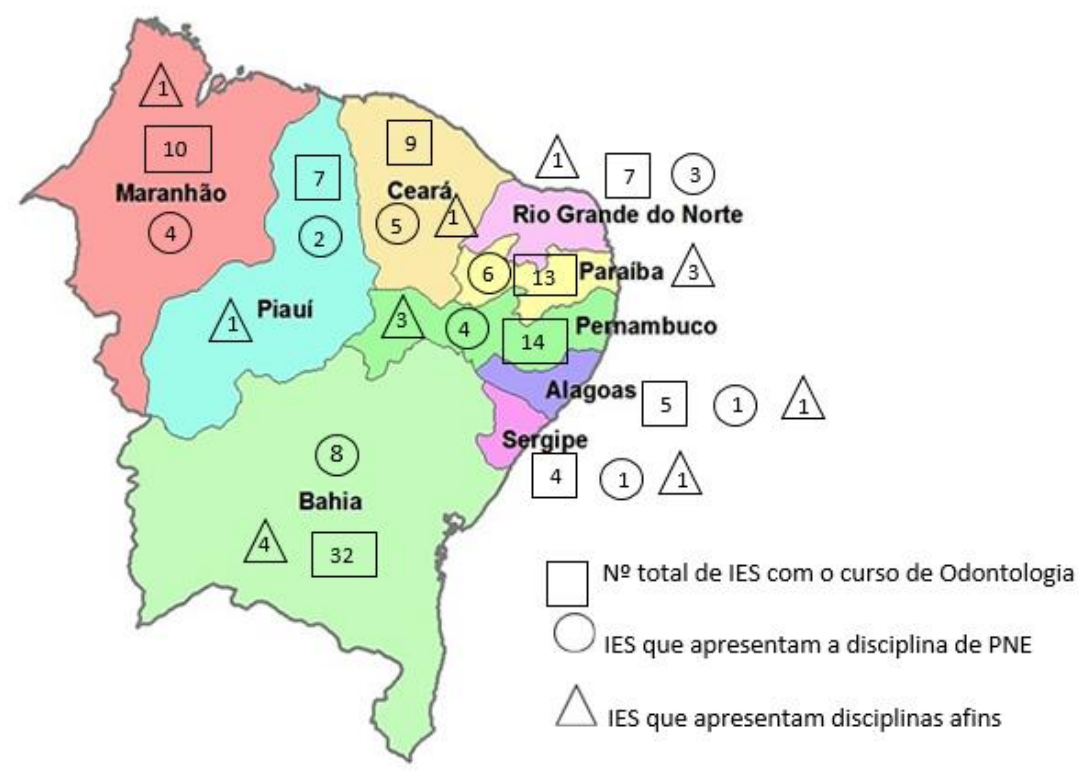

Figura 2. Mapa apresentando IES por estado e a oferta da disciplina de Odontologia para PNE.

Proporcionalmente ao total de IES por estado, Ceará e Paraíba são os que oferecem mais graduações de Odontologia com disciplina de PNE, cerca de 55,5\% e 46,1\% respectivamente das instituições ali localizadas. Em seguida: Rio Grande do Norte e Maranhão com 42,8\% e 40\% respectivamente; Pernambuco, 28,5\%; Piauí, 28,5\%; Bahia, 25\%; Alagoas, 20\%; e Sergipe com $25 \%$ (Tabela 1). 


\begin{tabular}{lcccc}
\hline \multicolumn{3}{l}{ Tabela 1. Classificação por estados e apresentação do componente. } \\
\hline $\begin{array}{c}\text { Estados do } \\
\text { Nordeste }\end{array}$ & \multicolumn{4}{c}{ Instituições de Ensino Superior } \\
\cline { 2 - 5 } & $\begin{array}{c}\text { Total (1) } \\
\mathbf{n}\end{array}$ & $\begin{array}{c}\text { Matriz } \\
\text { disponível } \\
\mathbf{n}(\%)\end{array}$ & $\begin{array}{c}\text { Não apresentam } \\
\text { a disciplina(2) } \\
\mathbf{n ~ ( \% ) ~}\end{array}$ & $\begin{array}{c}\text { Apresentam a } \\
\text { disciplina(2) } \\
\mathbf{n}(\%)\end{array}$ \\
\hline Alagoas & 5 & $5(100,0)$ & $4(80,0)$ & $1(20,0)$ \\
Bahia & 32 & $25(78,1)$ & $17(68,0)$ & $8(32,0)$ \\
Ceará & 9 & $9(100,0)$ & $4(44,4)$ & $5(55,5)$ \\
Maranhão & 10 & $5(50,0)$ & $1(20,0)$ & $4(80,0)$ \\
Paraíba & 13 & $9(69,2)$ & $3(33,3)$ & $6(66,6)$ \\
Pernambuco & 14 & $9(64,2)$ & $5(55,5)$ & $4(44,4)$ \\
Piauí & 7 & $4(57,1)$ & $2(50,0)$ & $2(50,0)$ \\
RG.do Norte & 7 & $6(85,7)$ & $3(50,0)$ & $3(50,0)$ \\
Sergipe & 4 & $4(100,0)$ & $3(75,0)$ & $1(25,0)$ \\
Total & $\mathbf{1 0 1}(\mathbf{1 0 0 )}$ & $\mathbf{7 6 ( 7 5 , 2 )}$ & $\mathbf{4 2}(\mathbf{5 5 , 2 )}$ & $\mathbf{3 4 4 4 , 7 )}$ \\
\hline
\end{tabular}

(1) Instituições de Ensino Superior cadastradas no e-MEC com o curso de Odontologia

(2) Componente exclusivo para pacientes portadores de necessidades especiais, na matriz curricular do curso de Odontologia

Quando avaliados novamente os currículos, em relação ao modo de oferta da disciplina, $91,1 \%$ apresentaram-se obrigatórias; $8,8 \%$ eletivas e $21 \%$ apresentam disciplinas afins.

\section{Discussão}

A presente pesquisa optou por busca na internet sobre o tema por considerar esse meio de comunicação mais ágil e com maior probabilidade de apresentar os dados buscados ${ }^{11}$.

Observa-se um avanço quanto ao debate da temática de Odontologia para PNEs na IES do Brasil. Uma pesquisa mostrou que 11 instituições contemplavam o componente de PNE, enquanto os dados do presente trabalho apontam, dentre os currículos disponíveis, 34 escolas de graduação apresentando ao menos uma disciplina exclusiva para Pacientes com Necessidades Especiais ${ }^{10}$.

Os resultados da atual pesquisa mostram a Bahia ainda com o maior número de disciplinas de PNE nos cursos de odontologia, obtendo 08 cursos, seguidos de Paraíba com 06, Ceará 05, Pernambuco e Maranhão com 04 em cada estado, Rio Grande do Norte com 03, Piauí 02, Sergipe e Alagoas com 01. 10 os estados que apresentaram o número maior de instituição com abordagem ao tema, foram a Bahia, Maranhão e Piauí, com 04, 02 e 02 respectivamente; Ceará, Paraíba, Pernambuco e Piauí e Rio Grande do Norte com 01 em cada, e Alagoas e Sergipe com nenhuma ${ }^{10}$. 
Ceará que nos resultados aparecia com 01 curso com a disciplina de PNE, agora mostra 05 cursos, o que pode estar relacionado à disponibilidade da matriz ou com a abertura de novo $\operatorname{cursos}^{10}$.

Os estados de Sergipe e Alagoas apresentam um número pequeno de IES com odontologia para PNE, apenas $01 \mathrm{em}$ cada, baseados na última pesquisa do IBGE, realizada em 201012, mostrando que além do Nordeste apresentar índice de $26,3 \%$ em prevalência de pessoas deficientes, maior que a média nacional $(23,9 \%)$, os estados de Sergipe e Alagoas ainda superam esses números apresentando taxas de 25,09\% e 27,54\% respectivamente, podendo isso ser resposta da acessibilidade dos habitantes aos recursos básicos, ou a falta dela, como educação e saúde, oferecidos em cada estado.

No Nordeste, segundo os dados da atual pesquisa, aproximadamente $91,1 \%$, ofertam a disciplina de odontologia para PNE durante a graduação e é disponível como obrigatória, ou seja, a maior parte das instituições que dispõe da disciplina a oferecem de modo obrigatório e 8,8\% é oferecida como optativa.

As IES do Brasil devem basear suas ideias de ensino no modelo de saúde fornecido no país, tornando os futuros dentistas, médicos e demais, inteirados e despertos para as sensibilidades e particularidades de cada grupo de pessoas e região².

O presente estudo almeja contribuir para despertar uma maior valorização da abordagem ao tema. Sugere-se, uma maior divulgação e ampliação de conhecimento por parte das IES, incluindo e tornando obrigatório(s) componente(s) curricular(es) teórico-prático que abordem o conteúdo de Odontologia para Pessoas com Necessidades Especiais aos alunos de graduação despertando um olhar mais inclusivo e mais acolhedor por todos.

Os estudantes de graduação, futuros cirurgiões-dentistas, devem ser instruídos como generalistas, portanto, mesmo que não tenham feito especialização de Odontologia para Pacientes com Necessidades Especiais devem estar condicionados a cuidar da saúde desse segmento populacional, sendo capacitados a detectar as doenças da cavidade oral e compreender suas relações sistêmicas para melhor diagnosticar, tratar e/ou encaminhar quando necessário ${ }^{13}$.

\section{Conclusão}

Menos da metade das IES do Nordeste brasileiro oferecem uma disciplina no curso de Odontologia exclusiva para Pacientes com Necessidades Especiais, onde, o modo de oferta é predominantemente obrigatório. 


\section{Referências}

1. Campos CDC, Frazão BB, Saddi GL, Morais LA, Ferreira MG, Setúbal PCO, Alcântara RT. Manual prático para o atendimento odontológico de pacientes com necessidades especiais. $2^{\mathrm{a}}$ ed, Goiânia, Universidade Federal de Goiás - Faculdade de Odontologia, 2009, 26-29.

2. Oliveira JS, Prado Júnior RR, Fernandes RF, Mendes RF. Promoção de saúde bucal e extensão universitária: novas perspectivas para pacientes com necessidades especiais. Revista da ABENO. 2015;15(1):63-69.

3. Silva DCN, Silva TM, Nogueira MS, Mendonça RMC, Valente PHF, Araújo RF, Brito FAV. Acessibilidade de Portadores de Deficiência Física ou Mobilidade Reduzida na Unidade Básica de Saúde Jonas Manoel Dias em São Luís de Montes Belos-GO. Revista Eletrônica Faculdade Montes Belos. 2016;8(3):36-60.

4. Brasil MS. Secretaria de Atenção à Saúde. Departamento de Ações Programáticas Estratégicas. Manual de legislação em saúde da pessoa com deficiência. 2.ed. Brasília: Editora do Ministério da Saúde, 2006.

5. Pereira LM, Mardero E, Ferreira SH, Floriani Kramer P, Barreto CR. Atenção odontológica em pacientes com deficiências: a experiência do curso de Odontologia da ULBRA Canoas/RS. Stomatos. 2010;16(31):92-99.

6. Castilho LS, Resende VLS, Barros ACP, Souza Lacerda DC, Marques EEM, Frias Castro N, Oliveira Pacheco A. Atendimento odontológico a pacientes com necessidades especiais: Considerações a respeito de um projeto de extensão. Revista ELO-Diálogos em Extensão. 2015; 2(1):16-32.

7. Ditterrich RG, Portero PP, Schmidt LM. A preocupação social nos currículos de odontologia. Rev. Abeno. 2007;7(1):58-62.

8. Fonseca ALA, Azzalis LA, Fonseca FLA, Botazzo C. Análise qualitativa das percepções de cirurgiões-dentistas envolvidos nos atendimentos de pacientes com necessidades especiais de serviços públicos municipais. Revista brasileira de crescimento e desenvolvimento humano. 2010; 20(2):208-216.

9. Gomes MJ, Caxias FP, Margon CD, Rosa RG, Carvalho RB. A percepção dos docentes do Curso de Odontologia da UFES em relação à necessidade de inclusão da disciplina denominada Atendimento Odontológico a Pacientes Portadores de Necessidades Especiais. Revista Brasileira de Pesquisa em Saúde. 2009; 11(1): 33-39.

10. Bonato LL, Lopes AMS, Silva CM, Itner RG, Silva ACH. Situação atual da formação para assistência de pessoas com necessidades especiais nas faculdades de odontologia no Brasil. Clínica e Pesquisa em Odontologia-UNITAU. 2013;5(1):10-15. 
11. Graziosi MES, Liebano RE, Nahas FX. Pesquisa em base de dados. Curso de especialização em Saúde da Família. São Paulo: UnaSUS/Unifesp. Recuperado em 19Dez2014 de http://www. unasus. unifesp. br/biblioteca_virtual/esf/1/modulo_cientifico/Unidade_13.pdf. 2013.

12. Oliveira LMB. "Cartilha do Censo 2010-Pessoas com deficiência." Brasília: SDH-PR/SNPD, 2012.

13. Peres AS, Peres SHCS, Silva RHA. Atendimento a pacientes especiais: reflexão sobre os aspectos éticos e legais. Rev Fac Odontol Lins. 2005;17(1):49-53. 